\title{
FOLLOW-UP OBSERVATIONS OF PTFO 8-8695: A 3 MYR OLD T TAURI STAR HOSTING A JUPITER-MASS PLANETARY CANDIDATE
}

\author{
David R. Ciardi ${ }^{1}$, Julian C. van Eyken ${ }^{1,2,3}$, Jason W. Barnes ${ }^{4}$, Charles A. Beichman ${ }^{1}$, Sean J. Carey ${ }^{5}$, \\ Christopher J. Crockett ${ }^{6}$, Jason Eastman ${ }^{3,7}$, Christopher M. Johns-Krull ${ }^{8}$, Steve B. Howell ${ }^{9}$, Stephen R. Kane ${ }^{10}$, \\ Jacob N . Mclane ${ }^{11}$, Peter Plavchan ${ }^{12}$, L. Prato ${ }^{11}$, John Stauffer ${ }^{5}$, Gerard T. van Belle ${ }^{11}$, and Kaspar von Braun ${ }^{11}$ \\ ${ }^{1}$ NASA Exoplanet Science Institute/Caltech, Pasadena, CA, USA; ciardi@ipac.caltech.edu \\ ${ }^{2}$ University of California, Santa Barbara/LCOGT, Santa Barbara, CA, USA \\ ${ }^{3}$ Las Cumbres Observatory Global Telescope Network, Goleta, CA, USA \\ ${ }^{4}$ University of Idaho, Moscow, ID, USA \\ ${ }^{5}$ Spitzer Science Center/Caltech, Pasadena, CA, USA \\ ${ }^{6}$ Science News, Washington, DC, USA \\ ${ }^{7}$ Harvard-Smithsonian Center for Astrophysics, Cambridge, MA, USA \\ ${ }^{8}$ Rice University, Houston, TX, USA \\ ${ }^{9}$ NASA Ames Research Center, Mountain View, CA, USA \\ ${ }^{10}$ San Francisco State University, San Francisco, CA, USA \\ ${ }_{11}$ Lowell Observatory, Flagstaff, AZ, USA \\ ${ }^{12}$ Missouri State University, Springfield, MO, USA \\ Received 2014 October 8; accepted 2015 June 29; published 2015 August 7
}

\begin{abstract}
We present Spitzer $4.5 \mu \mathrm{m}$ light curve observations, Keck NIRSPEC radial velocity observations, and LCOGT optical light curve observations of PTFO 8-8695, which may host a Jupiter-sized planet in a very short orbital period ( 0.45 days). Previous work by van Eyken et al. and Barnes et al. predicts that the stellar rotation axis and the planetary orbital plane should precess with a period of 300-600 days. As a consequence, the observed transits should change shape and depth, disappear, and reappear with the precession. Our observations indicate the longterm presence of the transit events ( $>3$ years), and that the transits indeed do change depth, disappear and reappear. The Spitzer observations and the NIRSPEC radial velocity observations (with contemporaneous LCOGT optical light curve data) are consistent with the predicted transit times and depths for the $M_{\star}=0.34 M_{\odot}$ precession model and demonstrate the disappearance of the transits. An LCOGT optical light curve shows that the transits do reappear approximately 1 year later. The observed transits occur at the times predicted by a straight-forward propagation of the transit ephemeris. The precession model correctly predicts the depth and time of the Spitzer transit and the lack of a transit at the time of the NIRSPEC radial velocity observations. However, the precession model predicts the return of the transits approximately 1 month later than observed by LCOGT. Overall, the data are suggestive that the planetary interpretation of the observed transit events may indeed be correct, but the precession model and data are currently insufficient to confirm firmly the planetary status of PTFO 8-8695b.
\end{abstract}

Key words: planetary systems - stars: individual (PTFO 8-8695, 2MASS J05250755+0134243, CVSO 30) stars: pre-main sequence

Supporting material: machine-readable tables

\section{INTRODUCTION}

With the discoveries fueled by the Kepler Mission (Borucki et al. 2010), there are now more than 1800 confirmed or validated planets (e.g., Batalha et al. 2011; Rowe et al. 2014). Kepler has increased our knowledge of the diversity of planets and planetary systems, and the sheer number of planets discovered by Kepler, coupled with on-going discoveries from other transit programs, radial velocity surveys, direct imaging efforts, and microlensing campaigns (e.g., Kraus \& Ireland 2012; Gillon et al. 2014; Koshimoto et al. 2014; Wittenmyer et al. 2014), have spawned a realization that nature yields planetary systems in a variety of configurations (e.g., Ciardi et al. 2013; Steffen 2013; Fabrycky et al. 2014; Winn \& Fabrycky 2014). To understand the processes that shape the planetary systems that we observe today, it is crucial to observe the planets during the period of formation and evolution.

Evidence has been found for extra-solar rings around a 16 Myr old pre-main-sequence star in the Upper Centaurus-Lupus subgroup of Sco-Cen, possibly indicative of early planet formation around a $\mathrm{T}$ Tauri star (Mamajek et al. 2012).
However, discovering and observing planets in the earliest stages of formation and evolution requires observing stars that are only $<5-10 \mathrm{Myr}$ old, as this is the timescale over which the planet forming disks are depleted (e.g., Haisch et al. 2001; Hillenbrand 2008), although recent work may indicate that the disks may last as long as <20 Myr (Pfalzner et al. 2014). However, stars at this age are notoriously active and spotted, making them photometrically and spectroscopically variable and making planet discovery at these young ages difficult (e.g., Miller et al. 2008; Crockett et al. 2012; Cody et al. 2014).

The Palomar Transient Factory (PTF) Orion survey, conducted in the winters of 2009 and 2010 (van Eyken et al. 2011, 2012), was an attempt to address this deficit by searching for young transiting hot Jupiters in the 25 Ori group, a small association of $\mathrm{T}$ Tauri stars identified by Briceño et al. (2005, 2007). In van Eyken et al. (2012), we reported the discovery of a promising young planetary candidate orbiting the known weak-lined T Tauri star PTFO 8-8695 with an age previously estimated of $\approx 3 \mathrm{Myr}$ (Briceño et al. 2005). Superimposed on top of notable quasi-periodic variability, the star 
showed regular transit events with a period of just 0.45 days. However, unlike TW Hya, which is a classical T Tauri star with active accretion (Rucinski \& Krautter 1983) and has periodic $\sim 2 \%$ dips likely caused by the disk occulting a hot spot (Siwak et al. 2014), PTFO 8-8695, being a WTTS, has no infrared excess and likely no disk to occult or veil the star. Finally, no evidence was found for a stellar companion.

The observed optical radial velocity variations (Keck HIRES and HET HRS) were found to be out of phase with the transit events and were not of sufficient amplitude to be caused by a stellar companion, arguing that the observed transits were not the result of an eclipsing binary (van Eyken et al. 2012). This is in contrast to V471 Tau which shows similar depth eclipses, but also $150 \mathrm{~km} \mathrm{~s}^{-1}$ amplitude radial velocity variations in phase with the eclipses caused by a stellar mass white dwarf orbiting a K-dwarf (Kamiński et al. 2007). The radial velocity variations observed in the optical for PTFO 8-8695 are likely caused by the rotation of the spotted stellar surface (Huélamo et al. 2008; Huerta et al. 2008; Prato et al. 2008). The spot induced observed radial velocity variations in the optical ( $\sim 2 \mathrm{~km} \mathrm{~s}^{-1}$ semi-amplitude) yield a candidate companion mass upper limit of $M \sin i<4.8 M_{\text {Jup }}$. Coupled with the transit modeling and the constraints on the line of sight inclination $\left(i \approx 60^{\circ}\right.$; van Eyken et al. 2012), the upper limit of the companion mass was found to be $M_{\mathrm{p}} \lesssim 5 M_{\text {Jup }}$-well within the planetary mass regime.

The transit light curves did show shape changes between the two years in which the PTF Orion observations were made. Barnes et al. (2013) showed that these effects could result from planetary transits of a fast-rotating, oblate, and significantly gravitationally darkened star, which is consistent with the short rotation period and high projected rotational velocity of PTFO 8-8685 ( $v \sin i \approx 80 \mathrm{~km} \mathrm{~s}^{-1}$; van Eyken et al. 2012). Being non-spherical, and darker at the equator than at the poles (von Zeipel 1924), oblique transits of such a rapidly rotating star can show unusual and asymmetric transit shapes (Barnes 2009). Furthermore, if the planet orbit is oblique to the stellar rotation axis, a torque is exerted on the orbit by the stellar oblateness, leading to precession of the orbital nodes.

Orbital precession has been noted previously in other systems and its effect on observed planetary transitsparticularly in circumbinary systems (e.g., Leung \& Lee 2013; Martin \& Triaud 2014). Recently, such behavior has previously been noted in the Kepler $13 \mathrm{~b}$ planet system (Barnes et al. 2011; Szabó et al. 2011, 2012, 2014), and with the circumbinary planet, Kepler 413b (Kostov et al. 2014). These are predicted to precess on timescales of decades. However, van Eyken et al. (2012) and Barnes et al. (2013) showed that, for PTFO 8-8695, the precession period was on the order of a 300-600 days - a timescale accessible to observation.

Barnes et al. (2013) were able to simultaneously fit both years of the PTF Orion data set using a fully consistent model which included gravitational darkening, oblateness of the host star, and an analytical model of the precession of the system. The fits yielded a planet mass $M_{\mathrm{p}} \sim 3 M_{\mathrm{Jup}}$, and predicted rapid precession on approximately year-long timescales. The fits implied a very high obliquity for the orbit $\left(\approx 70^{\circ}\right)$, resulting in a prediction that the transits should change depth, shape, and disappear for periods of a few months to a year as the precession brings the planet's orbit out of the line of sight to the star. The limited timespan of the data and the uncertainty in the stellar mass and radius left an unresolved degeneracy in the solution. More observations were needed to test the veracity of the model and better determine the system parameters. In particular, observations were needed to test that the transits changed shape, disappeared, and reappeared in a predictable manner.

Toward this end, we obtained follow-up observations of PTFO 8-8695 which included Spitzer photometry to confirm the transit events in the infrared; Keck NIRSPEC infrared radial velocity measurements to measure the radial velocity signature of the planet and the Rossiter-McLaughlin effect (McLaughlin 1924; Rossiter 1924; Queloz et al. 2000; Gaudi \& Winn 2007) as the planet transited the star, hinted at in the original optical radial velocity data (van Eyken et al. 2012); optical photometric monitoring with the Las Cumbres Observatory Global telescope Network (LCOGT) to support the radial velocity measurements and to better establish the transit parameters and orbit.

The Spitzer data, the Keck NIRSPEC data, and early optical LCOGT photometry data were acquired before the realization that the transits could disappear completely, but these data, in the end, provided evidence for the changing and disappearing transits. Further optical LCOGT observations were obtained with the realization that the system could precess and that the transits could disappear. The optical data show the return of the transits at the time predicted by propagating the transit midpoint ephemeris, but approximately 1 month prior to the prediction of the precession model. Given the complexity of the system as described in van Eyken et al. (2012) and Barnes et al. (2013), we regard the partial matching of the observations to the model as an indication that the planetary interpretation of the nature of the source of the transits is still viable, but we are unable to confirm the planet with these data and the current models.

\section{OBSERVATIONS AND ANALYSIS}

\subsection{Spitzer $4.5 \mu \mathrm{m}$}

Spitzer Space Telescope Director's discretionary time was granted to observe PTFO 8-8695 on 2012 April 28 UT, soon after the discovery paper was published. The primary purpose of the observations was to confirm the presence of the transit two years after the PTF Orion data were acquired (van Eyken et al. 2012), and to search for a secondary eclipse. The infrared observations of PTFO 8-8695 consist of an $11.8 \mathrm{hr}$ stare at $4.5 \mu \mathrm{m}$ with IRAC (request key 45476608), consisting of 1368 thirty second integrations with an effective cadence of $\sim 31.3 \mathrm{~s}$. The data were collected using the standard techniques for highprecision photometry with IRAC to minimize instrumental systematics (Ingalls et al. 2012). The observations were placed on the part of an array pixel that has minimal response variations. The standard Spitzer Science Center data products were used in our analysis.

Aperture photometry was performed on each basic calibrated data (BCD) image using a three pixel radius aperture and subtracting a background using an estimate of the mode of a circular annulus with inner radius of 3 and outer radius of 7 . Source positions were determined on each BCD by calculating the center-of-light using a $5 \times 5$ pixel box centered on the peak pixel associated with the source using the Spitzer Science Center provided IDL procedure box centroid.pro; aperture photometry was performed utilizing the IDL version of the 
Table 1

Spitzer Light Curve Data (Sample)

\begin{tabular}{|c|c|c|c|c|c|c|}
\hline BJD & Phase & Flux & Uncertainty & Flux Model & Flux-Model & Transit Model \\
\hline 2456045.70197 & -0.0014 & 0.9912959 & 0.0032780 & 0.9981057 & -0.0068097 & -0.0058880 \\
\hline 2456045.70233 & -0.0006 & 0.9932295 & 0.0033324 & 0.9980942 & -0.0048646 & -0.0058900 \\
\hline 2456045.70269 & 0.0002 & 0.9914524 & 0.0037372 & 0.9980825 & -0.0066302 & -0.0058890 \\
\hline 2456045.70305 & 0.0010 & 0.9927249 & 0.0039651 & 0.9980709 & -0.0053461 & -0.0058860 \\
\hline
\end{tabular}

(This table is available in its entirety in machine-readable form.)
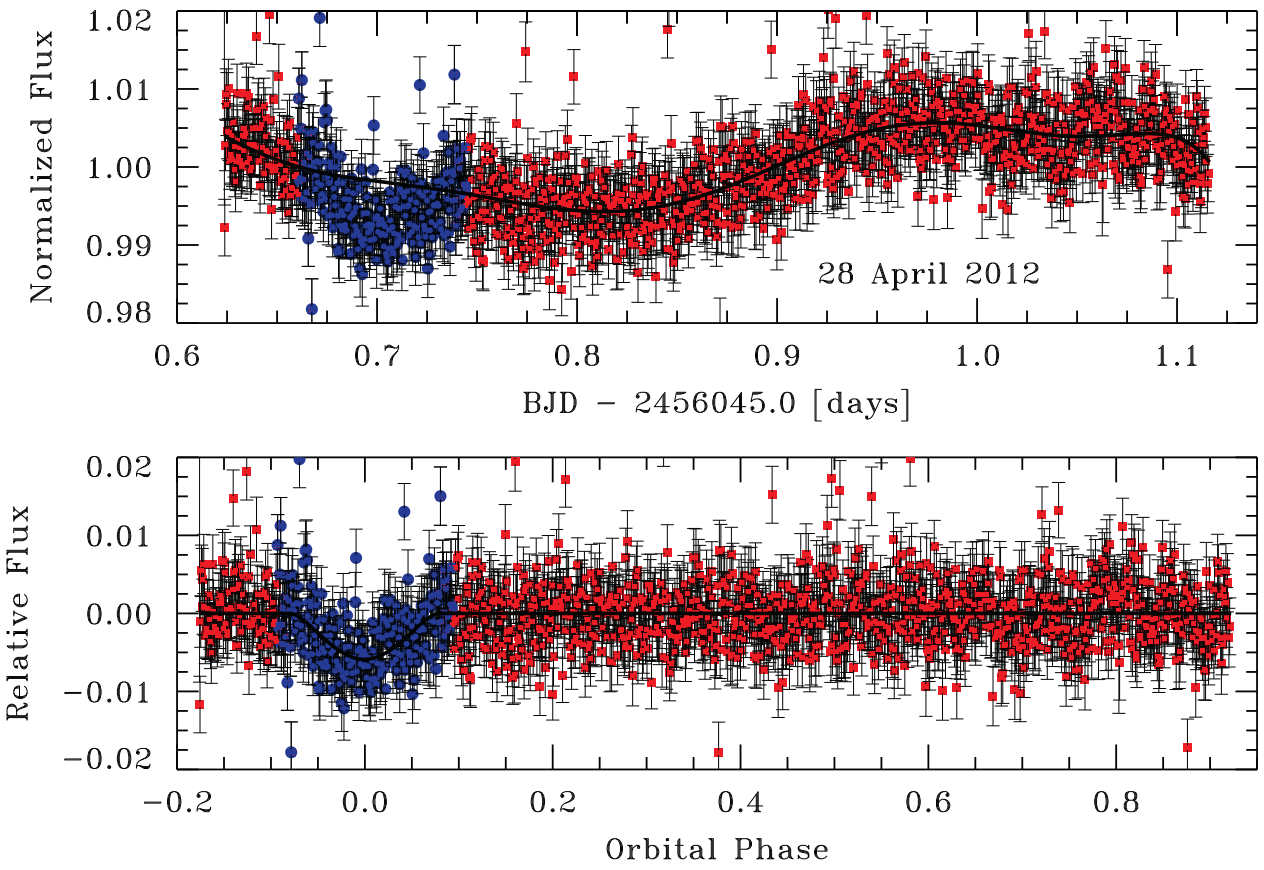

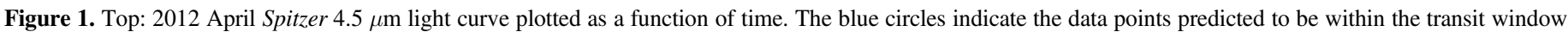

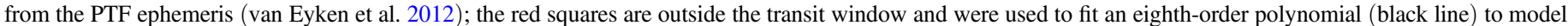

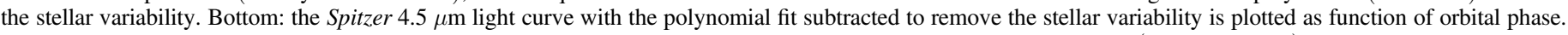
The solid black line represents the transit fit to the light curve and the data have been phased on this new ephemeris (see Section 3.1).

DAOPHOT routine aper.pro. Photometric systematics were removed by applying an intra-pixel gain map (Ingalls et al. 2012) to each photometric point as a function of centroid position. The Spitzer light curve is tabulated in Table 1 and shown in Figure 1.

\subsection{Keck-II NIRSPEC Radial Velocities}

Keck-II NIRSPEC observations were obtained on 2012 December 21-a full two years after the detection of the optical transits with PTF. The purpose of the NIRSPEC observations was to obtain phase-resolved high precision radial velocity data, primarily during the transit of PTFO 8-8685b. The Rossiter-McLaughlin effect was predicted to be $\approx 2-3 \mathrm{~km} \mathrm{~s}^{-1}$ given the $v \sin i$ of the star and the depth of the transit (van Eyken et al. 2012).

Spectra were acquired through the 0 ". 432 (3 pixel) wide slit; the echelle and cross disperser angles were oriented to obtain K-band spectra containing $\mathrm{NaI}, \mathrm{Mg}$ I and $\mathrm{CO}$. In this configuration, orders 32-38 (non-contiguous) were imaged through the NIRSPEC-7 blocking filter. The data were acquired in pairs of exposures of $600 \mathrm{~s}$ in a standard ABBA sequence, with the telescope being nodded $\pm 6^{\prime \prime}$ from the slit center along the $24^{\prime \prime}$ slit, so that each frame pair would contain object and sky in both nod positions.
To correct for telluric absorption, we also obtained spectra of HR 2315, an A0V star located close to PTFO 8-8695 in the sky, before and after the PTFO 8-8685 observations. After every two ABBA sets, spectra of the internal NIRSPEC continuum lamp were taken for flat fields at the K-band settings; lamp exposures of the argon, neon, krypton, and xenon arc lamps provided wavelength calibration for all the K-band orders. At the end of the PTFO 8-8695 science observations, GJ281, a radial velocity standard, was observed to set the absolute velocity scale of the observations. The spectral images were dark subtracted and flat fielded and the individual nods were extracted, wavelength calibrated with the lamp spectra, and telluric divided. Four ABBA nod sets were obtained for a total of 16 spectra and radial velocity estimates. The night was plagued with variable high cirrus and as a result, the signal-to-noise per resolution element varied but was approximately 50 .

The $\mathrm{Na}$ I lines at $\sim 2.206 \mu \mathrm{m}$ are the strongest lines in the near-infrared spectrum of PTFO 8-8695 and the entire order containing these lines was used to determine the relative radial velocities. The telluric corrected individual spectra were coadded to produce a master spectrum with signal-to-noise ratio 200 . The master spectrum was cross correlated with each of the 16 individual spectra to determine the relative radial 
Table 2

Keck NIRSPEC Radial Velocity Data

\begin{tabular}{lrcc}
\hline \hline HJD & Phase $^{\mathrm{a}}$ & Radial Velocity $\left(\mathrm{km} \mathrm{s}^{-1}\right)$ & Uncertainty $\left(\mathrm{km} \mathrm{s}^{-1}\right)$ \\
\hline 2456282.791 & -0.272 & -0.971 & 1.034 \\
2456282.805 & -0.241 & -0.098 & 1.082 \\
2456282.818 & -0.212 & 0.265 & 0.932 \\
2456282.832 & -0.181 & 0.364 & 1.000 \\
2456282.872 & -0.092 & 0.963 & 0.975 \\
2456282.886 & -0.060 & 0.890 & 0.902 \\
2456282.902 & -0.024 & -1.104 & 0.945 \\
2456282.916 & 0.007 & 1.398 & 0.881 \\
2456282.934 & 0.047 & -0.642 & 1.065 \\
2456282.948 & 0.078 & -0.456 & 2.327 \\
2456282.964 & 0.114 & 0.800 & 0.999 \\
2456282.977 & 0.143 & -0.114 & 1.046 \\
2456282.995 & 0.183 & -0.866 & 1.055 \\
2456283.009 & 0.214 & -0.743 & 1.798 \\
2456283.024 & 0.247 & -2.783 & 1.828 \\
2456283.038 & 0.279 & -1.003 & 1.167 \\
\hline
\end{tabular}

Note.

${ }^{\mathrm{a}}$ Based upon Spitzer Ephemeris (Table 4).

velocities between the spectra. A Monte Carlo effort was employed to estimate the uncertainties. The Monte Carlo simulations assume that the wavelength fits are perfect, which, of course, may not be correct. To try to characterize the wavelength fitting uncertainty, the spectra with strong telluric lines were utilized. The rms scatter in the radial velocity measurements of the telluric lines in each of the 16 spectra was found to be $0.68 \mathrm{~km} \mathrm{~s}^{-1}$. This uncertainty contribution was added in quadrature to the Monte Carlo uncertainty to arrive at a final uncertainty. The measured radial velocities and associated uncertainties are presented in Table 2, and the radial velocity curve is shown in Figure 2.

\subsection{LCOGT Photometry}

We obtained optical photometry for PTFO 8-8695 using the LCOGT $1 \mathrm{~m}$ telescope network during 2012 December and 2013 November. We utilized the full network capabilities of LCOGT including the sites at Cerro Tololo Inter-American Observatory in Chile (CTIO), Siding Spring Observatory in Australia (SSO), the South African Astronomical Observatory at Sutherland, South Africa (SAAO), and McDonald Observatory in Texas (MDO). The 2012 December data were obtained in support of the Keck NIRSPEC observations and were intended to be simultaneous with the Keck Observations, but poor weather prevented data from being obtained on the exact night of observations. Instead, contemporaneous data were obtained on 2012 December 23 UT. Exposure times were 90 s, resulting in a cadence of approximately $103 \mathrm{~s}$. All exposures were taken in the $r^{\prime}$ filter, similar to the filter used in the PTFOrion survey data.

After Barnes et al. (2013) published the precession model, it was recognized that the orbital plane could precess causing the transits to change, disappear and reappear, LCOGT was utilized again on 2013 November 09 UT to observe PTFO 88695 data in order to confirm the reappearance of the transit events and, in conjunction with the Spitzer photometry and the Keck radial velocity data, confirm the precession model and the planetary nature of PTFO 8-8695b. The 2013 November data were also obtained in the $r^{\prime}$ filter with an exposure time of
$120 \mathrm{~s}$; because the network of telescopes was able to observe the target with multiple telescopes at the same time, the effective cadence of the observations ranged from $\approx 2$ to $\approx 130$ $\mathrm{s}$ with the majority $(\approx 50 \%)$ of the observations obtained at a 130 s cadence.

In order to create the differential photometry, an initial normalization curve was created by taking the simple mean in magnitude space of all the raw reference light curves. For each reference star light curve, the mode of the residuals against the normalization curve was then subtracted, so that all the reference star light curves were normalized to the same flux level. Exposure by exposure, the mode of all the nownormalized reference star magnitudes was found, yielding the differential offset correction needed for each exposure. These differential corrections were subtracted from the raw target light curve to produce the final differentially corrected photometry. The same corrections applied to the original reference stars themselves (which should yield flat light curves) provided an internal consistency check. For a more detailed overview of the differential photometry technique, see van Eyken et al. (2011). The LCOGT light curves are tabulated in Table 3 and shown in Figures 3 and 4.

\section{DISCUSSION}

\subsection{Transit Ephemerides}

The Spitzer light curve was acquired $\sim 502$ days past the time of the 2010 PTF transit midpoint determination; in that time, the planet candidate PTFO 8-8695b would have orbited its host star 1119 times. As a result, the timing of the transit in the Spitzer data was uncertain by 65 minutes. The transit was identified by visual inspection of the light curve at the time predicted by the PTF ephemeris. The transit event was found to be within the uncertainties of the predictions, but to locate the transit more precisely, we excluded those data within $\sim 2.5 \mathrm{hr}$ of the predicted transit time (Figure 1 blue circles) and fit an eighth-order polynomial to the data outside the transit window (Figure 1 red squares). Given the large number of data points in the Spitzer light curve outside the transit window (1174) and the fact that the polynomial is only intended to parametrically model the stellar variability, the order of the polynomial was set by requiring a non-significant change in the normalized variance of the residuals. The polynomial fit was subtracted from the Spitzer light curve (data in the transit window included) to produce a whitened light curve suitable for a transit model fit (bottom of Figure 1).

The Spitzer light curve was fit using EXOFAST (Eastman et al. 2013) which yielded a value and uncertainties via MCMC modeling for the Spitzer transit midpoint and the transit depth (see Table 4). The Spitzer transit was found to be 17 minutes earlier than predicted, well within the 65 minutes uncertainty from the 2010 PTF ephemeris uncertainty propagated to the time of the 2012 Spitzer observations. Thus, more than 16 months after the PTF observations, the transit event occurred close to the predicted time. However, the transit, unlike the optical PTF transits, was found to be only $\sim 0.6 \%$ deep.

One of the primary purposes of the Spitzer observations was to search for a secondary eclipse; however, we found no evidence for a secondary eclipse to within the limits of the data. At a semimajor axis distance of $0.00838 \mathrm{AU}$ from the star $\left(T_{\text {eff }}=3470 \mathrm{~K}, R_{\star}=1.4 R_{\odot}\right)$, the planet candidate should have an equilibrium temperature of $T_{\mathrm{eq}} \approx 800-1800 \mathrm{~K}$, 

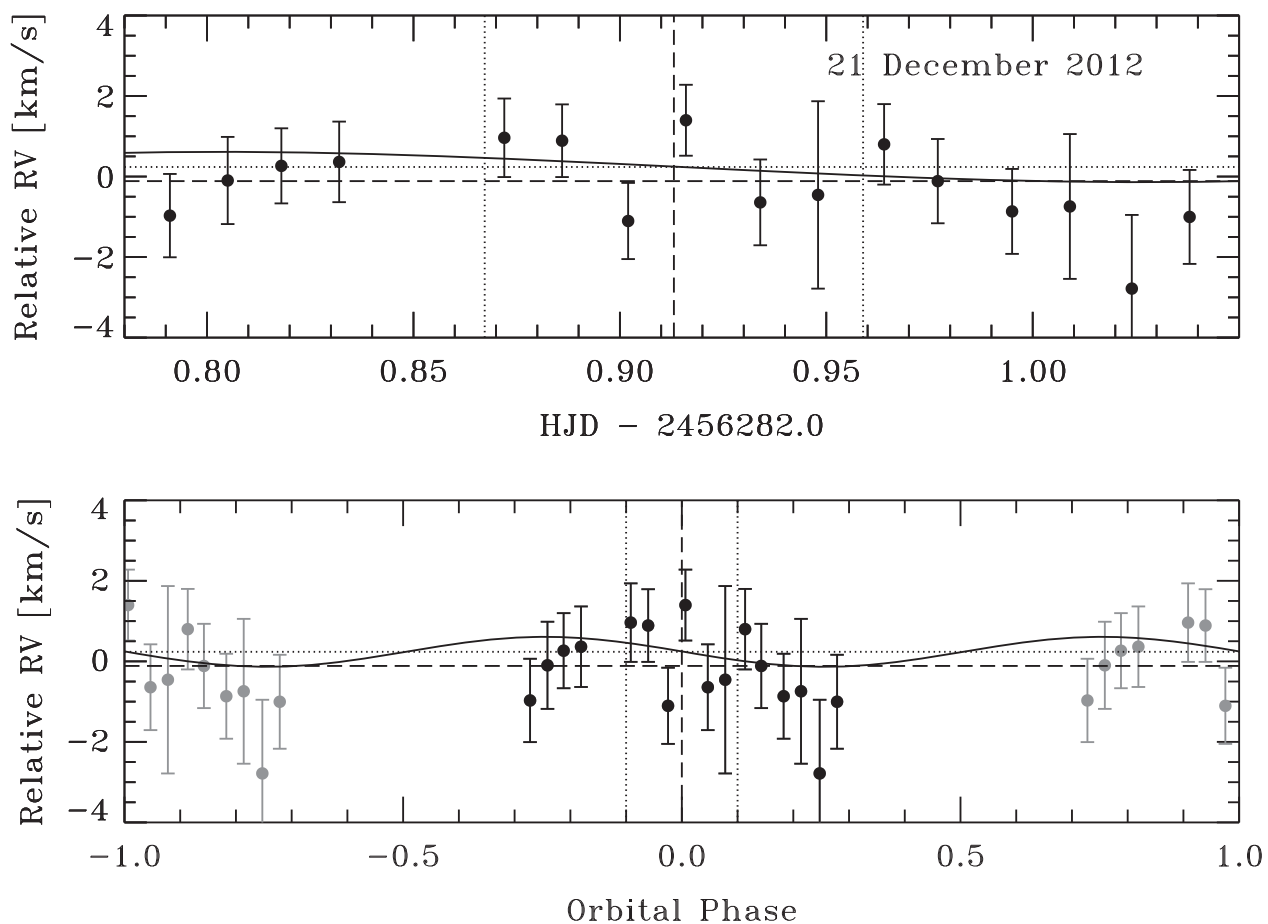

Figure 2. Top: 2012 December Keck NIRSPEC radial velocity data plotted as a function of time. The horizontal dashed line represents the weighted mean of the relative velocities and the solid black line represents the best fit radial velocity curve with the transit midpoint (vertical dashed line) fixed by the Spitzer ephemeris (Section 3.1); the horizontal dotted line represents the velocity offset of the fit $\left(\gamma=0.237 \pm 0.415 \mathrm{~km} \mathrm{~s}^{-1}\right)$. The vertical dotted lines represent the predicted beginning and ending times of ingress and egress, respectively. Bottom: the radial velocity curve is phased on the Spitzer ephemeris. The overplotted lines represent the fits as in the top panel; the gray points are the values repeated in phase for continuity and clarity.

Table 3

LCOGT Light Curve Data (Sample)

\begin{tabular}{|c|c|c|c|c|c|c|}
\hline BJD & Phase & Flux & Uncertainty & Flux Model & Flux-Model & Transit Model \\
\hline \multicolumn{7}{|l|}{$2012 \mathrm{Dec}$} \\
\hline 2456284.54447 & -0.4014 & 1.0143089 & 0.0052217 & 1.0215716 & -0.0072627 & $\cdots$ \\
\hline 2456284.54568 & -0.3987 & 1.0232391 & 0.0051967 & 1.0214167 & 0.0018223 & $\cdots$ \\
\hline 2456284.54687 & -0.3960 & 1.0129543 & 0.0051269 & 1.0212653 & -0.0083110 & $\cdots$ \\
\hline 2456284.54807 & -0.3933 & 1.0188872 & 0.0050400 & 1.0211184 & -0.0022312 & $\ldots$ \\
\hline \multicolumn{7}{|l|}{2013 Nov } \\
\hline 2456605.75391 & -0.0035 & 0.9584972 & 0.0040311 & 0.9926078 & -0.0341105 & -0.0272040 \\
\hline 2456605.75483 & -0.0014 & 0.9682513 & 0.0047093 & 0.9919295 & -0.0236782 & -0.0273680 \\
\hline 2456605.75546 & 0.0000 & 0.9703735 & 0.0040732 & 0.9914676 & -0.0210941 & -0.0274150 \\
\hline 2456605.75639 & 0.0021 & 0.9600239 & 0.0046811 & 0.9907754 & -0.0307514 & -0.0273890 \\
\hline
\end{tabular}

(This table is available in its entirety in machine-readable form.)

depending upon the albedo of the planet-similar to the expected effective temperature of a few million year old Jupiter-mass planet (Baraffe et al. 2003). The Spitzer $4.5 \mu \mathrm{m}$ light curve places a limit on the depth of the secondary eclipse of $\lesssim 0.3 \%(3 \sigma)$. This translates to an upper limit on the planetary candidate radius of $R_{\mathrm{p}} \lesssim 1.5 R_{\mathrm{Jup}}$, in reasonable agreement with the radius derived directly from the transit depths.

The 2012 December LCOGT optical light curve was obtained 8 months after the Spitzer light curve, in support of the Keck radial velocity data. In that time, the candidate planet PTFO 8-8695b would have orbited an additional 530 times and have a predicted transit time uncertainty of $\sim 10$ minutes $(0.02$ in phase). As with the Spitzer light curve, data within $\sim 2.5 \mathrm{hr}$ of the predicted transit midpoint were excluded, and the out-oftransit data points were fit with a cubic spline; the flux brightening event that resembles a flare at BJD $=2456284.625$ prevented the use of a polynomial to parameterize the out-oftransit variability (Figure 3). After subtraction of the spline fit, the light curve shows no clear sign of a transit event to a limit of $\sim 0.7 \%(1 \sigma)$ within 0.02 in phase of the predicted transit time. Structure is seen throughout the residual light curve at all phases and is likely the result of correlated noise in the data and/or true variability in the star.

The 2013 November LCOGT optical light curve was obtained 560 days after the Spitzer light curve, as part of an effort to re-detect the transits-a necessary link in our efforts to confirm the orbital precession model. In that time, the the planetary candidate would have orbited an additional 1249 times and have a predicted transit time uncertainty of about 71 minutes. Even without the formal searching, the transit event is clearly seen at the time of predicted transit (see Figure 4). To be consistent with our analysis of the Spitzer light curve and the previous LCOGT light curve, data within 

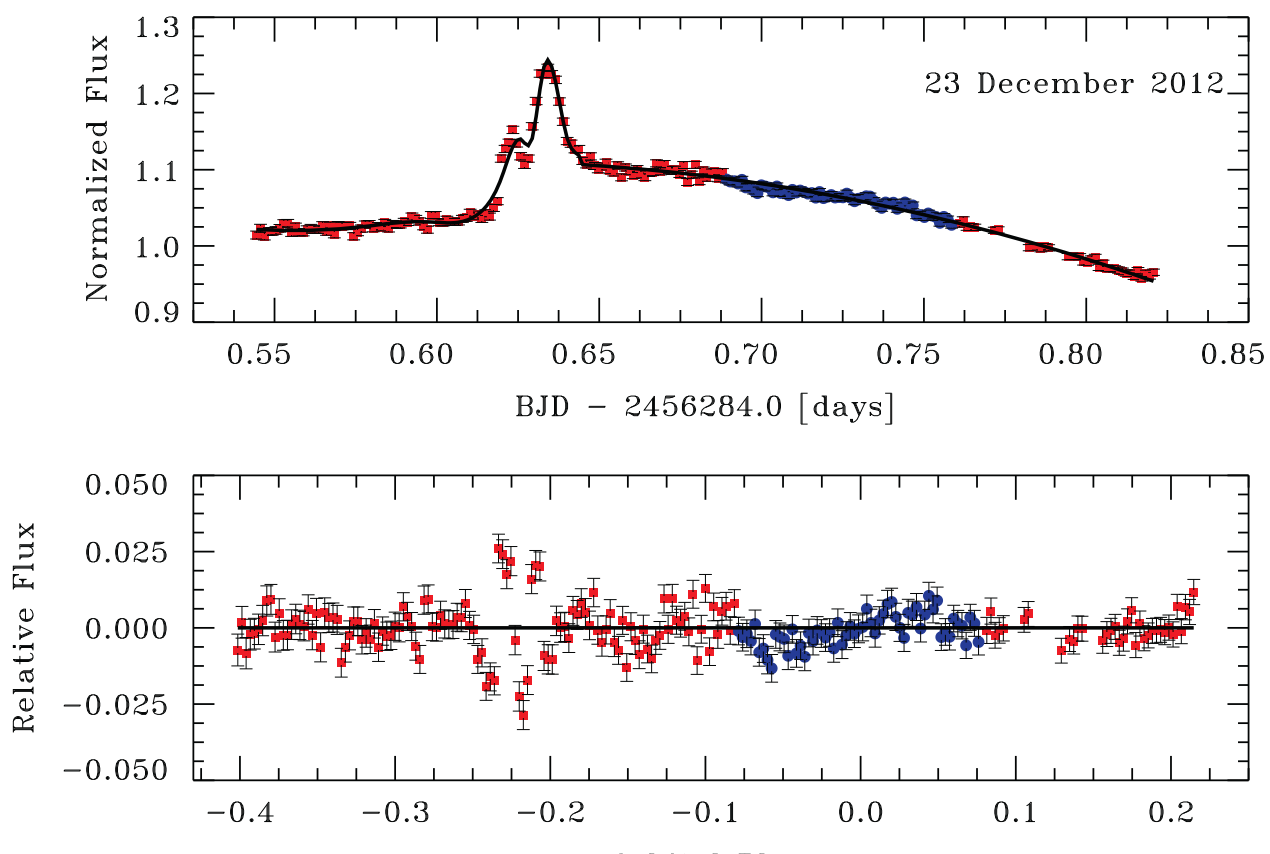

Figure 3. Top: 2012 December LCOGT $r^{\prime}$ light curve, obtained two days after the Keck NIRSPEC observations, is plotted as a function of time. The blue circles indicate the data points predicted to be within the transit window from the Spitzer ephemeris (Table 4); the red squares are outside the transit window and were used to fit a cubic spline (black line) to model the stellar variability. Bottom: the LCOGT $r^{\prime}$ light curve, phased upon the Spitzer ephemeris and with the spline fit subtracted, shows no sign of a transit. The solid black line represents a constant value of 0.0.


Figure 4. Top: 2013 November LCOGT $r^{\prime}$ light curve, obtained approximately 3 years after the PTF data and 1.5 years after the Spitzer data, is plotted as a function of time. The blue circles indicate the data predicted to be within the transit window from the Spitzer ephemeris (Table 4); the red points are outside the transit window and were used to fit a eighth-order polynomial (black line) to model the stellar variability. Bottom: the LCOGT light curve, with the polynomial fit subtracted to remove the stellar variability, is plotted as a function of orbital phase. The solid black line represents the transit fit to the light curve and the data have been phased on this new ephemeris (see Table 4).

$\sim 2.5 \mathrm{hr}$ of the predicted transit window were excluded (Figure 4 blue circles) and an eighth-order polynomial was fit to the data outside the transit window (Figure 4 red squares). The polynomial fit was subtracted from the LCOGT light curve (including data within the transit window) to produce a whitened light curve suitable for a transit model fit (bottom of Figure 4).

The light curve was fit using EXOFAST which yielded a value for the LCOGT transit midpoint and the transit depth (see Table 4). The transit was found 21 minutes earlier than 
Table 4

Transit Ephemerides

\begin{tabular}{|c|c|c|c|}
\hline & $\mathrm{PTF}^{\mathrm{a}}$ & Spitzer & LCOGT \\
\hline Approximate Date of Data Collection & 2009 Dec and 2010 Dec & $2012 \mathrm{Apr}$ & 2013 Nov \\
\hline Transit Midpoint $[\text { Reduced BJD }]^{\mathrm{b}}$ & 5543.9402 & 6045.7026 & 6605.7555 \\
\hline Midpoint Uncertainty & \pm 0.0008 & \pm 0.0009 & \pm 0.0004 \\
\hline Days Past PTF Midpoint & $\cdots$ & 501.7622 & 1061.8149 \\
\hline Number of Orbits since PTF Midpoint ${ }^{c}$ & $\ldots$ & 1119 & 2368 \\
\hline Uncertainty in Transit Prediction from PTF Midpoint & $\cdots$ & \pm 65 minutes & \pm 136 minutes \\
\hline Number of Orbits since Spitzer Midpoint ${ }^{c}$ & $\cdots$ & $\cdots$ & 1249 \\
\hline Uncertainty in Transit Prediction from Spitzer Midpoint & $\cdots$ & $\cdots$ & \pm 71 minutes \\
\hline Measured Offset from Spitzer Midpoint Prediction & $\cdots$ & $\cdots$ & -21.8 minutes \\
\hline
\end{tabular}

Notes.

${ }^{a}$ From van Eyken et al. (2012), Barnes et al. (2013).

${ }^{\mathrm{b}}$ Reduced BJD $=$ BJD-2450000.

${ }^{\mathrm{c}}$ Period held constant at $P=0.448413 \pm 0.000040$ days.

predicted but again well within the 71 minute uncertainty from the Spitzer ephemeris uncertainty propagated to the time of the LCOGT observations; in fact, the measured time of transit was only 39 minutes earlier than what was predicted from the PTF ephemeris which was established 3 years and 2368 orbits earlier. Thus, the planetary candidate PTFO 8-8685b transited the star as predicted via simple extension of the transit timing ephemerides; this transit has a measured depth of $\sim 2.7 \%$.

As a final note on the stability of the transit ephemeris, the period was originally determined to within $3.5 \mathrm{~s}$ (van Eyken et al. 2012). If the measured transit midpoints for the PTF, Spitzer, and LCOGT light curves were perfect, the period uncertainty, propagated to the dates of the observations, would still produce an uncertainty greater than the measured offsets of the observed Spitzer and LCOGT transits. If the period was shorter by only $0.98 \mathrm{~s}$, the transits would have been predicted at the times observed, indicating the quality of the original measured ephemerides.

\subsection{Radial Velocity Limits}

When originally proposed, the 2012 December Keck NIRSPEC radial velocity observations were intended to measure the Rossiter-McLaughlin effect as the planet transited the star. The observations were timed to be centered on the transit midpoint, as predicted from the PTF ephemeris. The amplitude and shape of the Rossiter-McLaughlin effect depends heavily on the $v \sin i$ of the star, the size of the transiting planet, the orbital geometry of the system, and the viewing geometry from the Earth. We predicted that given the transit depth of $\sim 4 \%$ and a stellar $v \sin i \approx 80 \mathrm{~km} \mathrm{~s}^{-1}$, the amplitude of the Rossiter-McLaughlin effect should be $2-4 \mathrm{~km} \mathrm{~s}^{-1}$ above the nominal radial velocity curve.

From Figure 2, it is clear that the Rossiter-McLaughlin effect was not detected, nor was the radial velocity signature of the planetary candidate PTFO 8-8695b detected within the limits of the Keck NIRSPEC data. The radial velocity curve is consistent with a constant value with a reduced chi-squared $\chi^{2}=0.85$. The lack of detection of the Rossiter-McLaughlin effect is consistent with the LCOGT light curve from 2012 December which showed that there was not a transiting event at the time of the Keck observations.
We have fitted the radial velocities with a Keplerian orbit, where the orbital period was fixed to 0.448413 days from van Eyken et al. (2012), and the orbital eccentricity was set to 0.0 consistent with both van Eyken et al. (2012) and Barnes et al. (2013). The time of the inferior conjunction (i.e., transit) was fixed by the Spitzer ephemeris listed in Table 4. The Keplerian orbital solution to the radial velocity data in Table 2 used RVLIN, a partially linearized, least-squares fitting procedure described in Wright \& Howard (2009); parameter uncertainties were estimated using the BOOTTRAN bootstrapping routines described in Wang et al. (2012). The results of the fitting are plotted over top the observations in Figure 2. The Keplerian orbital fit does not improve significantly the fit over the constant weighted mean with only a reduced chi-squared of $\chi^{2}=0.80$, and the semi-amplitude of the fit is statistically consistent with a flat line $\left(K=0.37 \pm 0.33 \mathrm{~km} \mathrm{~s}^{-1}\right)$.

\subsection{Comparison to the Precession Models}

The mass of the host star PTFO 8-8695 was estimated originally from isochrone fitting (Briceño et al. 2005), but the data could not distinguish between models from Baraffe et al. (2010) and Siess et al. (2000); thus, leaving an ambiguity in the stellar mass of either $\sim 0.44 M_{\odot}$ (Baraffe et al. 2010) or $\sim 0.34 M_{\odot}$ (Siess et al. 2000). As a result, Barnes et al. (2013) developed a set of models for both stellar mass estimates and fit the observed PTF transits from 2009 to 2010 separately and simultaneously. The separate models described the expected rotational axis and orbital planet precession of the star and planet, and predicted the depth, shape, and times of the planetary transits for each of the stellar masses. Additionally, the precession modeling does take into account the wavelength dependence of the transits - in particular, the effects of weakened limb and gravity darkening at the wavelengths of the Spitzer observations (Barnes et al. 2013). The data presented here are compared to the two models from Barnes et al. (2013).

The higher stellar mass $\left(0.44 M_{\odot}\right)$ precession model predicts a precession period of 581 days with a 184 days stretch with no transits visible at all as a result of the orbit precessing out of the line of sight. Within the time that transits are visible, there is a double peak of deep transits separated by about 200 days. The 


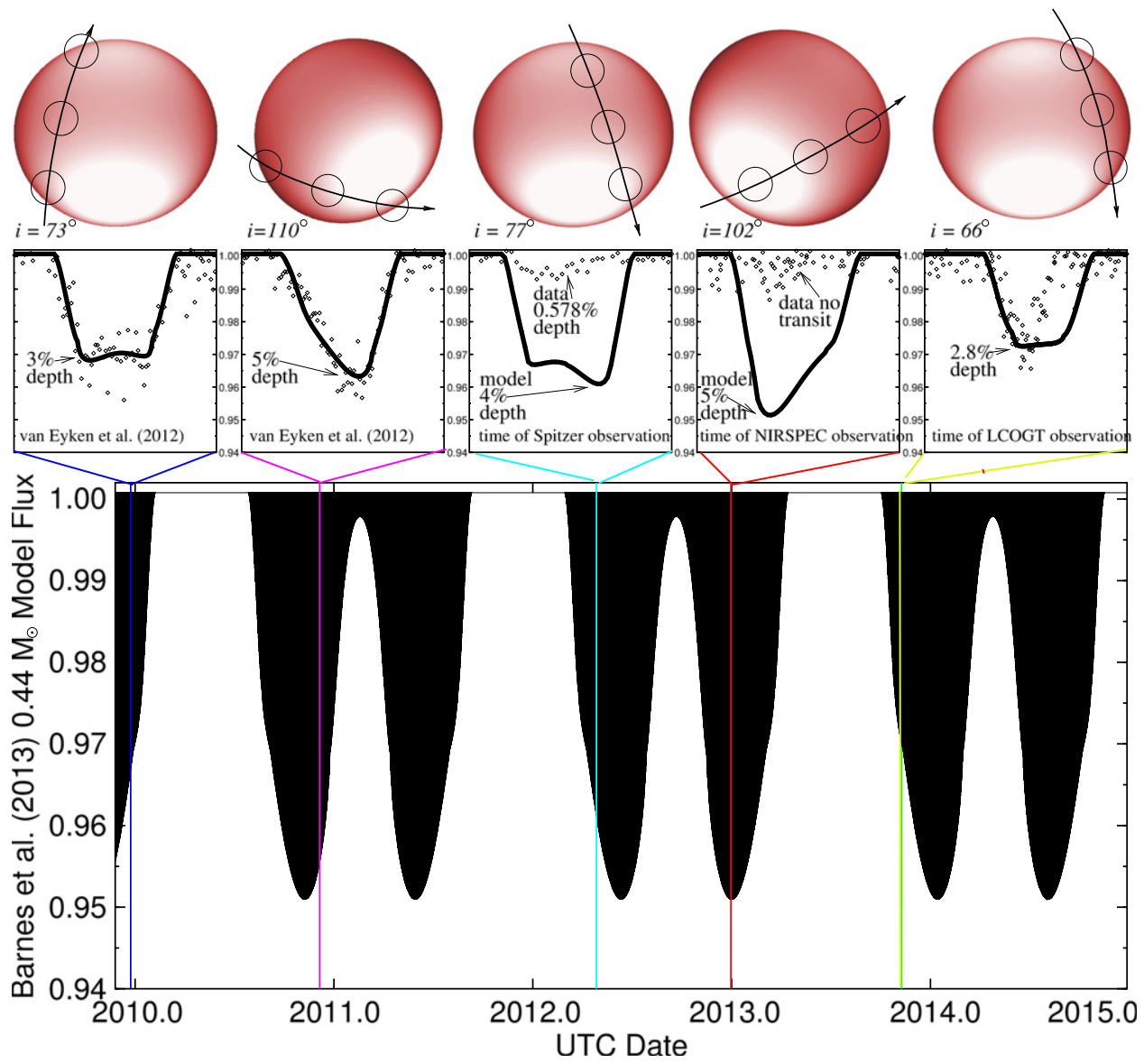

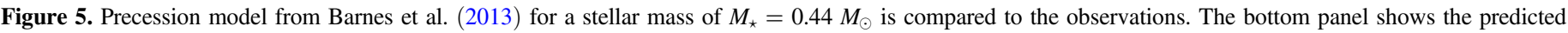

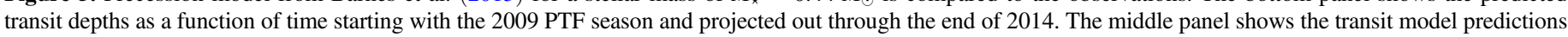

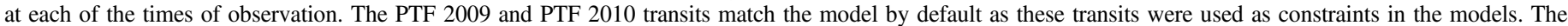

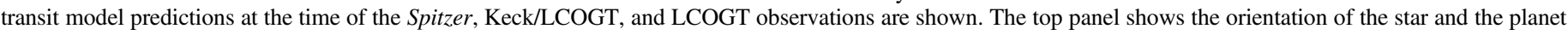

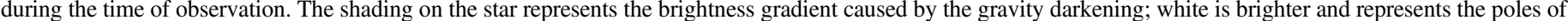

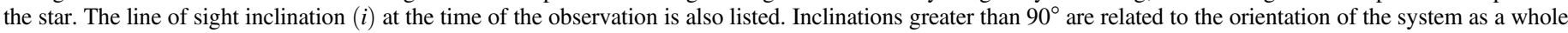
(for a description of the geometry see Barnes et al. 2013).

lower mass $\left(0.34 M_{\odot}\right)$ precession model predicts a similar transit structure as the higher mass model but on a more condensed time scale (Figure 6). The precession period is approximately 293 days and the time between the deep transits is about 80 days and the transits only disappear completely for approximately 75 days.

Overall, the observations do not agree well with the $0.44 M_{\odot}$ model. The Spitzer observations, observed to be only $\sim 0.6 \%$ in depth are predicted by the model to be $\sim 4 \%$. Further, the observations in 2012 December are predicted to have occurred when the transit depth was at its deepest $\sim 5 \%$. Such a deep 5\% deep transit would have been detected in the LCOGT data which have a transit detection limit of $\sim 0.7 \%$ (Figure 3 ). Additionally, with a line of sight inclination of $i=102^{\circ}$, the amplitude of the Rossiter-McLaughlin effect should have been $\gtrsim 2.5 \mathrm{~km} \mathrm{~s}^{-1}$ and would likely have been detected by the NIRSPEC radial velocity data at $>2 \sigma$. Finally, the 2013 November optical transit detection is predicted at approximately the right depth, but the shape of the observed transit is much narrower in comparison to the predicted transit, indicating that the model incorrectly represents the system orientation at the time of the observations. Thus, the $0.44 M_{\odot}$ precession model is inconsistent with the observations for each of the three epochs of data presented.
The $0.34 M_{\odot}$ precession model somewhat more closely matches the observations (Figure 4). The Spitzer light curve is represented well by the precession model transit prediction. Both the depth and the shape of the observed transit are in good agreement with the model prediction. Further, at the epoch of observations for the Keck NIRSPEC data and the first LCOGT light curve (2012 December), the model predicts that the system should be non-transiting, and, indeed, the observations support the disappearance of the transiting events. The model predicts the return of the transits in late 2013, and the 2013 November data confirm that the transits do reappear. However, the observations showed that the transits reappeared about 1 month earlier than predicted. With a precession orbital period of 293 days, the model is out-of-sync with the observations by $\sim 10 \%-15 \%$ of the predicted precession period.

The cause of the inconsistency of the $0.34 M_{\odot}$ model with the 2013 November observations, while predicting the 2012 April and December observations correctly, is difficult to discern, but could be the result of a variety of factors. The precession model depends heavily on knowledge of the stellar mass, the stellar moment of inertia, the stellar radius, and the planetary orbit eccentricity. The precession model assumes a circular orbit with a stellar radius of $\sim 1.04 R_{\odot}$; however, an eccentric orbit would allow for a larger stellar radius, which 


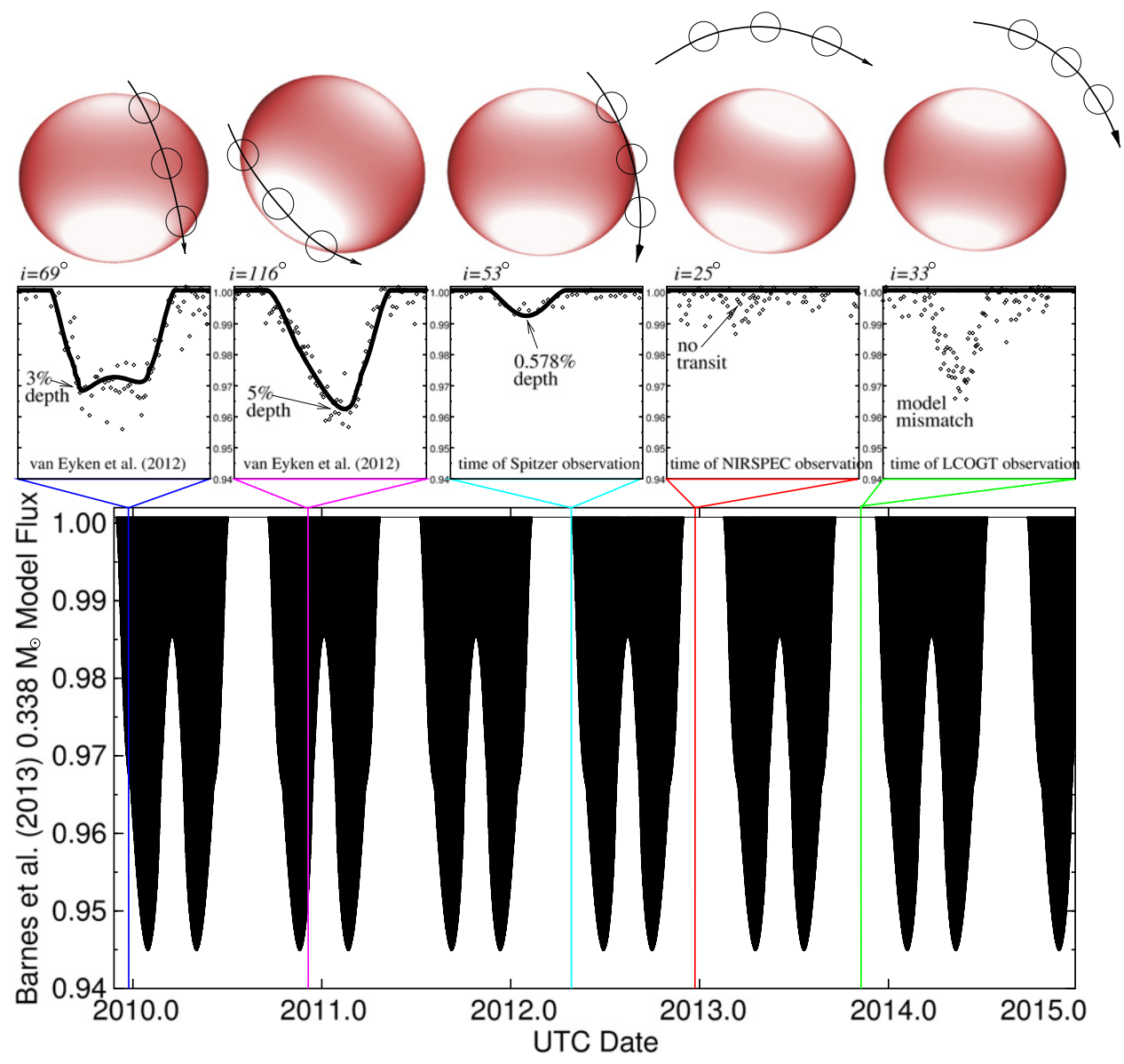

Figure 6. Precession model from Barnes et al. (2013) for a stellar mass of $M_{\star}=0.34 M_{\odot}$ is compared to the observations. The figure content is the same as for Figure 5.

would affect the details of the precession model and the precession period. The precession period is, to first order, proportional to the square of the stellar radius $\left(R_{\star}^{2}\right)$, and is also dependent on higher order powers of the stellar radius (e.g., $R_{\star}^{4}$; Murray \& Dermott 2000; Barnes et al. 2013); a 5\%-7\% change in the stellar radius could result in a $10 \%-15 \%$ change in the precession period.

The precession model also uses as inputs the orbital period and epochs of transits; even small errors on these, when propagated across three years and thousands of orbits, may explain why the $0.34 M_{\odot}$ model predicts correctly the 2012 data but not the 2013 data. Finally, the precession modeling fits not only the timing of the transits but also the shape of the transits. If spots have significantly altered the shape of the transit, the precession model may not correctly predict the system parameters - particularly when the model is propagated through thousands of orbits.

We cannot place better constraints on the stellar or planetary candidate parameters with the data presented here. The parameter space of the precession models is extremely large and is difficult to narrow and beyond the intended scope of this paper. A recent paper, studying the same system with our PTF data but without requiring the spin-orbit to be synchronously locked, find that a precession model can still reproduce the observations, with a precession period of 199 days and a planet mass of $\sim 4-5 M_{\text {Jup }}$ (Kamiaka et al. 2015). We are continuing to observe the planetary candidate with long-term photometric observations and radial velocity monitoring in an effort to assess more fully the existence and planetary nature of PTFO $8-8695 \mathrm{~b}$ and to more carefully determine the validity and parameters of the precession model.

While the observations presented here cannot uniquely identify which precession model might best describe the data, we can utilize the NIRSPEC radial velocity upper limits on the semi-amplitude to set limits on the potential mass of the planetary candidate. If the stellar mass is $0.44 M_{\odot}$, then the mass limit on a possible planetary candidate is $M_{\mathrm{p}} \sin i \lesssim 0.8 M_{\text {Jup }}$; if the stellar mass is closer to $0.34 M_{\odot}$, then the mass limit is $M_{\mathrm{p}} \sin i \lesssim 0.7 M_{\text {Jup }}$. As indicated by the data, the planet candidate does not transit during the time of the radial velocity observations, which sets an upper limit on the line of sight orbital inclination of $i \lesssim 50^{\circ}$. At that inclination, the planet candidate mass would have a mass of $M_{\mathrm{p}} \lesssim 0.9-1.1 M_{\text {Jup }}$. If the orbital inclination is as low as $i \sim 20^{\circ}-30^{\circ}$ as predicted by the $0.34 M_{\odot}$ precession model, then the planetary candidate could have a mass of $M_{\mathrm{p}} \lesssim 2.0-2.5 M_{\text {Jup }}$.

\subsection{Stellar Activity}

PTFO 8-8685 is a low mass young star and as a result is variable and is likely spotted as discussed in van Eyken et al. (2012; see also Koen 2015). The optical radial velocity curve modulations were attributed to the presence of spots, although not necessarily to the presence of a single spot. Large and longlived, high latitude spots are commonly present in weak-lined $\mathrm{T}$ 
Tauri stars (e.g., Stelzer et al. 2003; Rice et al. 2011), and spots have been seen to mimic eclipsing companions in other systems (e.g., RXJ1608.6-3922; Joergens et al. 2001). In that work, light curve data obtained in multiple filters and spectroscopic monitoring enabled the authors to determine that the deep eclipses $(0.5 \mathrm{mag})$, with long eclipse durations $(\sim 0.5$ in phase), were caused by stellar spots which disappeared in observations obtained 4 years later. They also found that the photometric variations were also consistent with the $2.4 \mathrm{~km} \mathrm{~s}^{-1}$ radial velocity variations but not compatible with an eclipsing binary.

van Eyken et al. (2012) could not find a spot distribution solution to fit the observed light curves for PTFO 8-8695, but we revisit the possibility of spots as a cause of the observed transit events here. Unlike RXJ1608, the observed transit features for PTFO 8-8695 are more shallow (3\%-5\%) and shorter in duration ( 0.1 in phase), and, as seen in 2009, sometimes flat-bottom during the event. In principle, a spotted surface with a single hot spot near the pole briefly eclipsed by the limb of the star may be able produce the short transit duration observed. However, such a hot spot would be expected to show a brightening $180^{\circ}$ out of phase with the flux dip but such a brightening is not seen in the data. In fact, many of the light curves from the 2009/2010 discovery data (van Eyken et al. 2012) display a brightness decrease near these phases in the light curves.

A cold spot below the equator could briefly come into view. In general, the spot size (i.e., the surface filling-factor) needed to produce the observed transit depth can be estimated if we assume a photospheric temperature to spot temperature ratio $\left(T_{\mathrm{p}} / T_{\mathrm{s}}\right)$. The star has an effective temperature of $T_{\mathrm{eff}} \approx 3470 \mathrm{~K}$ (Briceño et al. 2005) and M-dwarf spots can be $500-1000 \mathrm{~K}$ cooler than the photosphere (Barnes et al. 2011). The optical $(0.65 \mu \mathrm{m})$ detections of the transit event have depths that range from 3\%-5\% corresponding to spot filling factors of $\sim 3.5 \%-8 \%$. After taking into account the reduced contrast with the photosphere at longer wavelengths, the $0.58 \%$ infrared $(4.5 \mu \mathrm{m})$ transit depth corresponds to a filling-factor coverage of $\sim 1.5 \%-2.5 \%$ - a factor of $2-4$ times smaller. To explain the short transition duration of 0.1 in phase $\left(\sim 36^{\circ}\right.$ in longitude), such a spot would need to have a viewable longitudinal extent of $\sim 36 / 2 \sim 18^{\circ}$, and to yield a spot that covers $2 \%-8 \%$ of the surface, the viewable latitudinal extent of the spot would need to be $\sim 20^{\circ}-40^{\circ}$, but a spot of such latitudinal extent would likely be visible for more than just $1 / 10$ of the rotation period.

All of this is not impossible for an active low-mass star, but the transit events would need to change shape and depth (and be flat-bottomed as is the case for the 2009 data). At the same time, the spots would need to disappear and reappear at nearly the same stellar longitude over the course of $>3$ years. As indicated in Section 3.1, the transit times agree with the predicted ephemerides to within $\lesssim 20$ minutes or about $\lesssim 3 \%$ of the period. The spot would need to appear, evolve, disappear, and re-appear all within $\lesssim 10^{\circ}$ of the same stellar longitudinal position as the event timing ephemerides have been consistent for over three years, and do this all within the context of a generally spotted star producing the continuous variability of the star.

As in van Eyken et al. (2012), we find it difficult to model the transit events in a self-consistent manner with the data acquired over 3 years. We are, however, continuing to pursue multi-year and multi-color light curves in an effort to understand better the nature of the stellar variability.

In Section 3.1, we refer to the brightening event in the 2012 December optical light curve that occurs at phase 0.8 . This brightening does not look like a typical flare with a sharp rise and exponential decline (Walkowicz et al. 2011). One possibility is that this brightening is the result of an accretion event on the star. PTFO 8-8695 exhibits relatively strong $\mathrm{H} \alpha$ emission and it has been noted that the planetary candidate is near or at the Roche-limit-particularly, if the putative orbit is eccentric (van Eyken et al. 2012). As a result, the planetary candidate may be evaporating and the "flare" may actually be the result of infall onto the stellar surface. More detailed work on the $\mathrm{H} \alpha$ emission and its variability is the subject of another paper (C.M. Johns-Krull et al. 2015, in preparation).

\section{SUMMARY}

PTFO 8-8695b was discovered in the PTF-Orion survey for transiting exoplanets by van Eyken et al. (2012); PTFO $8-8695$ b was found to be in an 0.45 day orbit and to have a mass of $\lesssim 4-5 M_{\text {Jup }}$. That discovery was followed-up by a prediction by Barnes et al. (2013) that the stellar rotation axis and the orbital plane of the planet should precess and that the transits should change shape and depth and disappear and reappear with a period of 300-600 days. The two papers put together a coherent picture of a Jupiter-sized planetary candidate orbiting a $\sim 3 \mathrm{Myr}$ old weak-lined $\mathrm{T}$ Tauri star; however, the precession model needed confirmation and PTFO $8-8695$ b remained a planetary candidate with a mass less than $\lesssim 3 M_{\text {Jup }}$.

We have obtained follow-up observations of PTFO 8-8695 that includes Spitzer $4.5 \mu \mathrm{m}$ light curve observations, Keck NIRSPEC radial velocity observations, and LCOGT optical light curve observations. The data confirm that the transit events are persistent over many years and the times of the transits are predictable from the transit timing measurements, consistent with a steady period of 0.448 days. The transit events do appear to grow more shallow, disappear, and reappear as predicted by the precession modeling.

However, the precession model and observations are not in perfect agreement and more observations are needed to place better constraints on the model and to confirm the planetary nature of the candidate PTFO 8-9695b. Toward this end, we are pursuing additional long-term transit observations with LCOGT and radial velocity monitoring that will help limit the models, the orbit, the stellar parameters, and help confirm or refute the planetary nature as the source of the observed transit events which have remained for over three years of observations.

D.R.C. would like to dedicate this paper to his dad Robert A. Ciardi (1940-2013). Robert Ciardi had a passion for learning and knowledge and, in particular, for science. While not able to pursue a career in science for himself, he never stopped thinking and growing, and through his love and encouragement, D.R.C. was able to pursue his own love of exploration and science. In many ways, Robert Ciardi was more excited about this discovery than the authors on this paper. He will be greatly missed. Thank you, Dad. Some of the data presented herein were obtained at the W. M. Keck Observatory, which is operated as a scientific partnership among the California Institute of Technology, the University of California and the 
National Aeronautics and Space Administration. The Observatory was made possible by the generous financial support of the W. M. Keck Foundation. The authors wish to recognize and acknowledge the significant cultural role and reverence that the summit of Mauna Kea has always had within the indigenous Hawaiian community. We are most fortunate to have the opportunity to conduct observations from this mountain. This research has made use of the LCOGT Archive, which is operated by the California Institute of Technology, under contract with the Las Cumbres Observatory Global Telescope Network. This work is based, in part, on observations made with the Spitzer Space Telescope, which is operated by the Jet Propulsion Laboratory, California Institute of Technology under a contract with NASA. This research has made use of the NASA Exoplanet Archive, which is operated by the California Institute of Technology, under contract with the National Aeronautics and Space Administration under the Exoplanet Exploration Program. Portions of this work were performed at the California Institute of Technology under contract with the National Aeronautics and Space Administration.

\section{Facilities: Spitzer (IRAC), Keck:II (NIRSPEC), LCOGT.}

\section{REFERENCES}

Baraffe, I., Chabrier, G., \& Barman, T. 2010, RPPh, 73, 016901

Baraffe, I., Chabrier, G., Barman, T. S., Allard, F., \& Hauschildt, P. H. 2003, A\&A, 402, 701

Barnes, J. R., Jeffers, S. V., \& Jones, H. R. A. 2011, MNRAS, 412, 1599

Barnes, J. W. 2009, ApJ, 705, 683

Barnes, J. W., Linscott, E., \& Shporer, A. 2011, ApJS, 197, 10

Barnes, J. W., van Eyken, J. C., Jackson, B. K., Ciardi, D. R., \& Fortney, J. J. 2013, ApJ, 774, 53

Batalha, N. M., Borucki, W. J., Bryson, S. T., et al. 2011, ApJ, 729, 27

Borucki, W. J., Koch, D., Basri, G., et al. 2010, Sci, 327, 977

Briceño, C., Calvet, N., Hernández, J., et al. 2005, AJ, 129, 907

Briceño, C., Hartmann, L., Hernández, J., et al. 2007, ApJ, 661, 1119

Ciardi, D. R., Fabrycky, D. C., Ford, E. B., et al. 2013, ApJ, 763, 41

Cody, A. M., Stauffer, J., Baglin, A., et al. 2014, AJ, 147, 82

Crockett, C. J., Mahmud, N. I., Prato, L., et al. 2012, ApJ, 761, 164

Eastman, J., Gaudi, B. S., \& Agol, E. 2013, PASP, 125, 83

Fabrycky, D. C. 2014, ApJ, 790, 46

Gaudi, B. S., \& Winn, J. N. 2007, ApJ, 655, 550
Gillon, M., Anderson, D. R., Collier-Cameron, A., et al. 2014, A\&A, 562, L3 Haisch, K. E., Jr., Lada, E. A., \& Lada, C. 2001, AJ, 121, 2065

Hillenbrand, L. A. 2008, PhST, 130, 014024

Huélamo, N., Figueira, P., Bonfils, X., et al. 2008, A\&A, 489, L9

Huerta, M., Johns-Krull, C. M., Prato, L., Hartigan, P., \& Jaffe, D. T. 2008, ApJ, 678, 472

Ingalls, J. G., Krick, J. E., Carey, S. J., et al. 2012, Proc. SPIE, 8442, 84421 Y Joergens, V., Guenther, E., Neuhäuser, R., Fernández, M., \& Vijapurkar, J. 2001, A\&A, 373, 966

Kamiaka, S., Masuda, K., Xue, Y., et al. 2015, PASJ, in press (arXiv:1506.04829)

Kamiński, K. Z., Ruciński, S. M., Matthews, J. M., et al. 2007, AJ, 134, 1206 Koen, C. 2015, MNRAS, 450, 3991

Koshimoto, N., Udalski, A., Sumi, T., et al. 2014, ApJ, 788, 128

Kostov, V. B., McCullough, P. R., Carter, J. A., et al. 2014, ApJ, 784, 14

Kraus, A. L., \& Ireland, M. J. 2012, ApJ, 745, 5

Leung, G. C. K., \& Lee, M. H. 2013, ApJ, 763, 107

Mamajek, E. E., Quillen, A. C., Pecaut, M. J., et al. 2012, AJ, 143, 72

Martin, D. V., \& Triaud, A. H. M. J. 2014, A\&A, 570, A91

McLaughlin, D. B. 1924, ApJ, 60, 22

Miller, A. A., Irwin, J., Aigrain, S., Hodgkin, S., \& Hebb, L. 2008, MNRAS, 387,349

Murray, C. D., \& Dermott, S. F. 2000, Solar System Dynamics (New York: Cambridge Univ. Press)

Pfalzner, S., Steinhausen, M., \& Menten, K. 2014, ApJL, in press (arXiv:1409.0978)

Prato, L., Huerta, M., Johns-Krull, C. M., et al. 2008, ApJL, 687, L103

Queloz, D., Eggenberger, A., Mayor, M., et al. 2000, A\&A, 359, L13

Rice, J. B., Strassmeier, K. G., \& Kopf, M. 2011, ApJ, 728, 69

Rossiter, R. A. 1924, ApJ, 60, 15

Rowe, J. F., Bryson, S. T., Marcy, G. W., et al. 2014, ApJ, 784, 45

Rucinski, S. M., \& Krautter, J. 1983, A\&A, 121, 217

Schneider, J. 1994, P\&SS, 42, 539

Siess, L., Dufour, E., \& Forestini, M. 2000, A\&A, 358, 593

Siwak, M., Rucinski, S. M., Matthews, J. M., et al. 2014, MNRAS, 444, 327

Steffen, J. H. 2013, MNRAS, 433, 3246

Stelzer, B., Fernández, M., Costa, V. M., et al. 2003, A\&A, 411, 517

Szabó, G. M., Pál, A., Derekas, A., et al. 2012, MNRAS, 421, L122

Szabó, G. M., Simon, A., \& Kiss, L. L. 2014, MNRAS, 437, 1045

Szabó, G. M., Szabó, R., Benkő, J. M., et al. 2011, ApJL, 736, L4

van Eyken, J. C., Ciardi, D. R., Rebull, L. M., et al. 2011, AJ, 142, 60

van Eyken, J. C., Ciardi, D. R., von Braun, K., et al. 2012, ApJ, 755, 42 von Zeipel, H. 1924, MNRAS, 84, 684

Walkowicz, L. M., Basri, G., Batalha, N., et al. 2011, AJ, 141, 50

Wang, S. X., Wright, J. T., Cochran, W., et al. 2012, ApJ, 761, 46

Winn, J. N., \& Fabrycky, D. C. 2014, ARA\&A, in press (arXiv:1410.4199)

Wittenmyer, R., Horner, J., Tinney, C. G., et al. 2014, ApJ, 783, 103

Wright, J. T., \& Howard, A. W. 2009, ApJS, 182, 205 